\title{
Micrasterias (Zygnematophyceae) de duas áreas do Pantanal dos Marimbus (Baiano e Remanso), Chapada Diamantina, Bahia, Brasil
}

\section{Camila dos Anjos Ribeiro ${ }^{a}$, Geraldo José Peixoto Ramos ${ }^{b}$, Ivania Batista de Oliveira ${ }^{c}$, Carlos Wallace do Nascimento Moura*}

Laboratório de Ficologia, Departamento de Ciências Biológicas, Universidade Estadual de Feira de Santana, Bahia, Brasil

\begin{abstract}
Resumo - Foi realizado o inventário do gênero Micrasterias (Zygnematophyceae) em duas áreas alagáveis do Pantanal dos Marimbus (Baiano e Remanso), Chapada Diamantina, Bahia, como contribuição ao conhecimento da desmidioflórula do Estado. Os materiais estudados, de origem perifítica e metafítica, provieram de 120 unidades amostrais coletadas durante o período seco (abril, junho e agosto de 2011) e chuvoso (outubro e dezembro de 2011 e fevereiro de 2012). Foram registrados 15 táxons, sendo 11 comuns a ambos os Marimbus. Os táxons mais frequentes no período seco e no período chuvoso foram M. pinnatifida (Marimbus do Baiano) e M. truncata var. pusilla (Marimbus do Remanso). Com exceção de M. laticeps var. laticeps, 14 táxons tiveram sua distribuição geográfica ampliada para a Chapada Diamantina. Micrasterias tropica var. tropica e M. simplex var. minor são adições à flora ficológica do Nordeste do Brasil.

Palavras-chave adicionais: algas continentais, biodiversidade, desmídeas, pantanal.
\end{abstract}

\begin{abstract}
Micrasterias (Zygnematophyceae) from two areas of the Pantanal dos Marimbus (Baiano and Remanso), Chapada Diamantina, Bahia State, Brazil) - The genera Micrasterias (Zygnematophyceae) was surveyed in two wetland areas of Pantanal dos Marimbus (Baiano and Remanso), Chapada Diamantina, Bahia State, Brazil, as a contribution to the knowledge of desmid flora from the state. The periphytic and metaphytic materials came from 120 samples collected in dry (April, June and August 2011) and rainy (October and December 2011, and February 2012) seasons. Fifteen taxa were identified, 11 of which are common to both Marimbus areas. The most frequent taxa, both in dry and wet seasons, were M. pinnatifida (Marimbus do Baiano) and M. truncata var. pusilla (Marimbus do Remanso). Except for M. laticeps var. laticeps, 14 taxa had their geographical distribution expanded to the Chapada Diamantina. Micrasterias tropica var. tropica and M. simplex var. minor are additions to the desmid flora of northeastern Brazil.
\end{abstract}

Additional key words: biodiversity, desmids, freshwater algae, wetlands.

Micrasterias C.Agardh ex Ralfs (Zygnemaphyceae) compreende um dos 36 gêneros da família Desmidiaceae (Bicudo \& Menezes 2006). Devido às elevadas dimensões de algumas espécies (atingindo ca. $400 \mu \mathrm{m}$ compr.) e à sua forma geralmente recortada e simétrica, este grupo atrai a atenção, sendo utilizado em estudos sobre a morfogênese das semicélulas, mudanças na pressão de turgor, nucleação e poliploidia (Bicudo \& Sormus 1982; Guiry \& Guiry 2014). Várias espécies de Micrasterias são cosmopolitas, habitando geralmente o plâncton ou perifíton de ambientes aquáticos ácidos (lagoas, poças, lagos, pântanos, charcos) oligotróficos ou distróficos (Gerrath 2003).

Trabalhos taxonômicos de microalgas continentais realizados na Bahia ainda são escassos, apesar do Estado possuir uma grande diversidade de ecossistemas aquáticos e uma considerável extensão geográfica. Para Micrasterias, as informações estão restritas a Förster (1964), Martins \& Bicudo (1987), Bicudo \& Martins (1989), Oliveira et al. (2009), Oliveira (2011), Ramos et al. (2011) e Santana (2011), que ao todo listaram 41 táxons. Com exceção de

\footnotetext{
*Autor para correspondência: wallace@uefs.br;

${ }^{\mathrm{a}}$ caribeiro.bio@gmail.com; ${ }^{\mathrm{b} g e r a l d o j p r @ g m a i l . c o m ; ~}$

civboliveira@gmail.com

Editor responsável: Alessandro Rapini

Submetido em: 16 set. 2014; aceito em: 8 jan. 2015

Publicação eletrônica: 28 jan. 2015; versão final: 2 fev. 2015
}

Förster (1964), que registraram Micrasterias laticeps Nordst. var. laticeps, M. arcuata Bail. var. perforata K.Förster \& F.Eckert e M. arcuata var. subpinnatifida West \& G.S.West para o Pico das Almas e Rio de Conta, e de Ramos et al. (2011), que registraram $M$. crux-melitensis Ralfs e $M$. furcata C.Agardh ex Ralfs var. furcata f. furcata para a Serra da Jiboia, os demais trabalhos se restringiram à planície litorânea.

$\mathrm{O}$ presente trabalho apresenta o inventário do gênero Micrasterias em duas áreas do Pantanal dos Marimbus (Remanso e Baiano), Chapada Diamantina, Bahia, contribuindo para o conhecimento da desmidioflórula do Estado.

\section{MAterial e MÉtodos}

O Pantanal dos Marimbus, localizado na porção leste do Parque Nacional da Chapada Diamantina, possui relevo plano com uma grande área marginal sujeita a inundações periódicas devido à confluência dos rios Santo Antônio, Utinga e São José (Lima \& Nolasco 1997; Funch 2002; França et al. 2010; SEMA 2011). Este é subdividido em quatro áreas (Remanso, Fazenda Velha, Ferreira e Baiano) interligadas pelo rio Santo Antônio (Figura 1).

O presente estudo foi realizado em duas áreas do Pantanal dos Marimbus (Remanso e Baiano). O 
Marimbus do Remanso (12 $\left.40^{\circ} \mathrm{S}, 41^{\circ} 20^{\prime} \mathrm{W}\right)$, localizado no município de Lençóis e próximo à Vila de Remanso, é caracterizado por apresentar águas calmas (na maioria das suas zonas), com um pH de 6,6-7,6 e transparência moderada $(2,1 \mathrm{~m})$. O rio Santo Antônio tem uma notável influência sobre a dinâmica do meio ambiente, especialmente em suas partes mais estreitas, onde a correnteza é mais elevada. Já o Marimbus do
Baiano ( $\left.12^{\circ} 45^{\prime} \mathrm{S}, 41^{\circ} 18^{\prime} \mathrm{W},\right)$, localizado no município de Andaraí, consiste em várias lagoas interligadas pelo rio Santo Antônio: Lagoa da Isca, Lagoa dos Paus, Olho D'Agua do Peri e Lagoa do Baiano. As amostragens no Marimbus do Baiano foram realizadas na Lagoa do Baiano, caracterizada por apresentar águas calmas, pH 6,0-7,2 e transparência moderada $(0,9 \mathrm{~m})$ (Ramos et al. 2012, 2014).
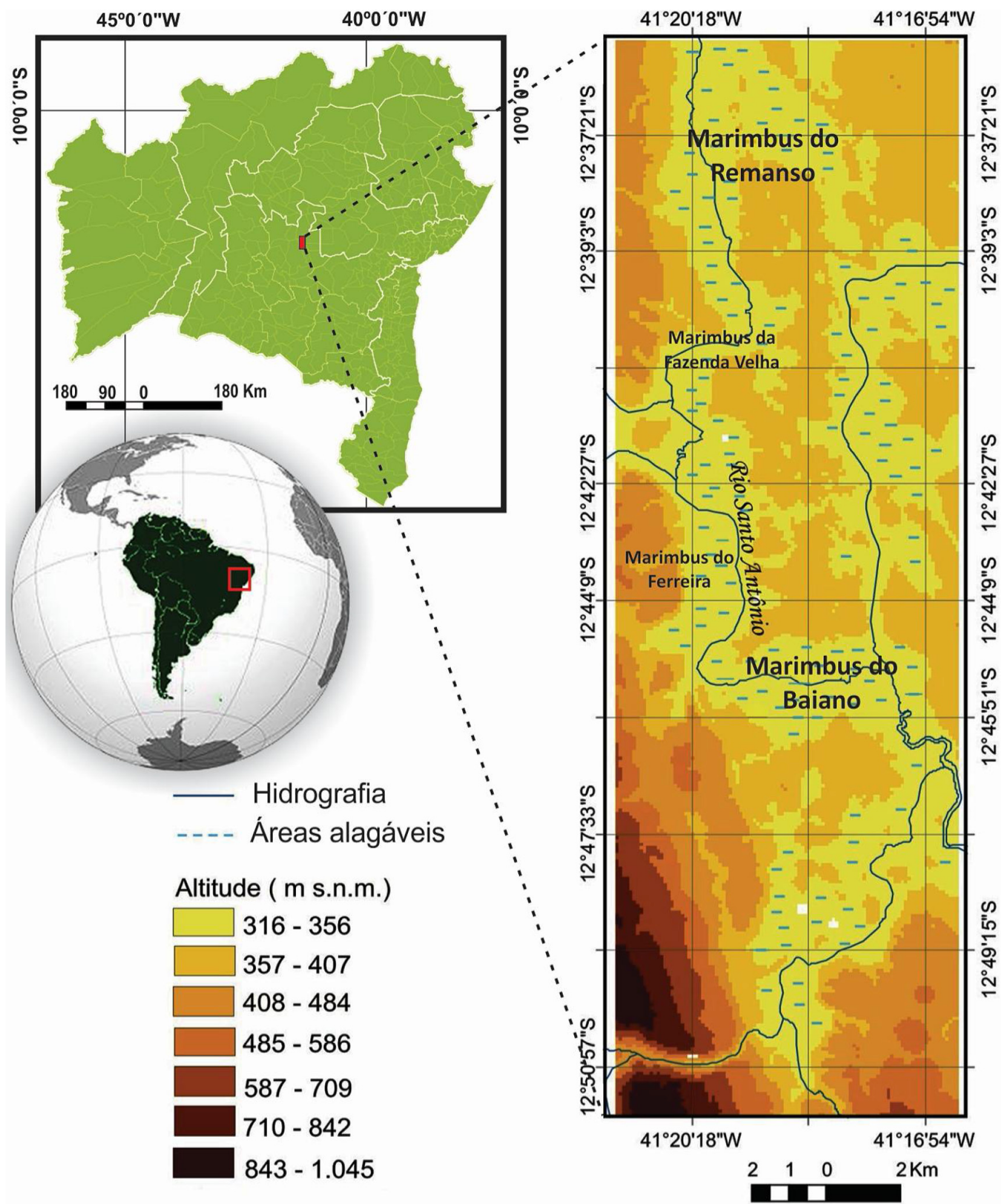

Figura 1. Mapa de localização do Pantanal dos Marimbus, APA Marimbus-Iraquara, Chapada Diamantina, Bahia, Brasil (baseado em Ramos et al. 2014). 
No Pantanal dos Marimbus ocorrem várias macrófitas, como Utricularia foliosa L., Cabomba haynesii Wiersema, Eichhornia azurea (Sw.) Kunth., Nymphaea Ampla (Salisb.) DC, Nymphoides hydrocleys (Humb. \& Bonpl. ex Willd.) e Salvinia auriculata Aubl.), que propiciam o desenvolvimento de algas perifíticas e metafíticas (Ramos et al. 2012).

O material foi coletado durante o período seco (abril, junho e agosto de 2011) e chuvoso (outubro e dezembro de 2011 e fevereiro de 2012), totalizando 120 unidades amostrais. As coletas foram realizadas segundo os métodos usuais empregados nos estudos de taxonomia de microalgas continentais (Bicudo \& Menezes 2006).

Os limites métricos das populações foram aferidos através de ocular micrométrica acoplada ao microscópio óptico e as identificações dos táxons baseadas em literatura especializada. As microfotografias foram obtidas com câmara fotográfica digital acoplada ao microscópio fotônico.

A frequência de ocorrência dos táxons inventariados foi calculada de acordo com a fórmula: $\mathrm{F}=\mathrm{n} .100 / \mathrm{N}$; onde, $\mathrm{n}=$ número de amostras em que uma espécie foi registrada; $\mathrm{N}=$ total de amostras analisadas. As categorias de frequência foram determinadas de acordo com Matteucci \& Colma (1982), tendo sido estabelecidas as seguintes categorias: $>70 \%$ - Muito Frequente $(\mathrm{MF}) ; \leq 70 \%$ e $>40 \%$ - Frequente $(\mathrm{F}) ; \leq$ $40 \%$ e $>10 \%$ - Pouco Frequente (PF); $\leq 10 \%$ - Rara (R).

As amostras foram preservadas em solução de Transeau preparada conforme Bicudo \& Menezes (2006) e tombadas na coleção líquida do Herbário da Universidade Estadual de Feira de Santana (HUEFS).

\section{RESULTADOS}

O inventário do gênero Micrasterias no Pantanal dos Marimbus (Remanso e Baiano) registrou 15 táxons, dos quais 14 tiveram a sua distribuição geográfica ampliada para a Chapada Diamantina. Dentre eles, Micrasterias tropica var. tropica e $M$. simplex var. minor são adições para a flora ficológica do Nordeste do Brasil.

\section{Tratamento TAXONÔMICO}

Micrasterias abrupta West \& G.S.West var. abrupta, Trans. Linn. Soc. Lond., ser. 2, 5(5): 241; pl. 14, fig. 13-16. 1896.

Figura 2.

Célula 46,3-50 $\mu \mathrm{m}$ compr., 47,5-60 $\mu \mathrm{m}$ larg., istmo 6,3-8,8 $\mu \mathrm{m}$ larg., lobo polar 26,3-31,3 $\mu \mathrm{m}$ larg.

Material examinado - BRASIL. BAHIA: Andaraí, Marimbus do Baiano, 1 abr. 2011, C.W.N. Moura \& G.J.P. Ramos s.n. (HUEFS 178327); ib., 29 jun. 2011, C.A. Ribeiro \& G.J.P. Ramos s.n. (HUEFS 178372); Lençóis, Marimbus do Remanso, 2 abr. 2011, C.W.N. Moura \& G.J.P. Ramos s.n. (HUEFS 178358).
Registros para o estado da Bahia. Oliveira (2011).

Morfologicamente próxima a $M$. truncata (Corda) Bréb. ex Ralfs, que difere por apresentar dimensões menores e lobos polares com margem côncava. As populações analisadas concordam com a descrição, medidas e ilustrações apresentadas por Bicudo \& Sormus (1982) para São Paulo e Oliveira (2011) para a APA Litoral Norte na Bahia.

Micrasterias alata G.C.Wall var. alata, Annls. Mag. Nat. Hist., ser. 3, 5(28): 279; pl. 13, fig. 11. 1860 Figura 3.

Célula 205-245 $\mu \mathrm{m}$ compr., 180-225 $\mu \mathrm{m}$ larg., istmo 15-25 $\mu \mathrm{m}$ larg., lobo polar 75-125 $\mu \mathrm{m}$ larg

Material examinado - BRASIL. BAHIA: Andaraí, Marimbus do Baiano, 1 abr. 2011, C.W.N. Moura \& G.J.P. Ramos s.n. (HUEFS 178332); ib., 29 jun. 2011, C.A. Ribeiro \& G.J.P. Ramos s.n. (HUEFS 178375); Lençóis, Marimbus do Remanso, 2 abr. 2011, C.W.N. Moura \& G.J.P. Ramos s.n. (HUEFS 178358)

Registros para o estado da Bahia. Oliveira et al. (2009) e Oliveira (2011).

Difere das demais espécies estudadas por apresentar semicélulas 3-lobadas, com lóbulos que, morfologicamente, lembram asas. Prescott et al. (1977) relataram um acentuado polimorfismo em $M$. alata, quanto à espessura dos lobos polares e laterais e às incisões interlobares, que podem ser ora profundas, ora mais superficiais. As populações estudadas, embora estejam de acordo com a descrição e ilustrações apresentadas por Oliveira et al. (2009) e Oliveira (2011), diferem por possuir dimensões maiores.

Micrasterias foliacea Bailey ex Ralfs var. foliacea, Brit. Desm.: 210; pl. 35, fig. 3. 1848.

Figuras 4 e 5.

Célula 77-87,5 $\mu \mathrm{m}$ compr., 85-100 $\mu \mathrm{m}$ larg., istmo 13-17,5 $\mu \mathrm{m}$ larg., lobo polar 35-45 $\mu \mathrm{m}$ larg.

Material examinado - BRASIL. BAHIA: Andaraí, Marimbus do Baiano, 1 abr. 2011, C.W.N. Moura \& G.J.P. Ramos s.n. (HUEFS 178327); Lençóis, Marimbus do Remanso, 10 dez. 2011, C.A. Ribeiro \& G.J.P. Ramos s.n. (HUEFS 185407).

Registros para o estado da Bahia. Oliveira et al. (2009) e Oliveira (2011).

É facilmente identificada por apresentar talo filamentoso, nos quais as células estão unidas pelo imbricamento de espinhos dos lobos polares. Segundo Prescott et al. (1977), estes filamentos podem ser constituídos por até 182 células.

Micrasterias furcata C.Agardh ex Ralfs var. furcata $\mathrm{f}$. furcata, Brit. Desm.: 73; pl. 9, fig. 2. 1848.

Figura 6 e 7.

Célula 150-180 $\mu \mathrm{m}$ compr., 125-170 $\mu \mathrm{m}$ larg., istmo 12,4-20 $\mu \mathrm{m}$ larg., lobo polar 43,7-75 $\mu \mathrm{m}$ larg.

Material examinado - BRASIL. BAHIA: Andaraí, Marimbus do Baiano, 1 abr. 2011, C.W.N. Moura \& G.J.P. Ramos s.n. (HUEFS 178332); ib., 29 jun. 2011, C.A. Ribeiro \& G.J.P. Ramos s.n. (HUEFS 178375), 9 dez. 2011, C.A. Ribeiro \& G.J.P. Ramos s.n. (HUEFS 185395). 

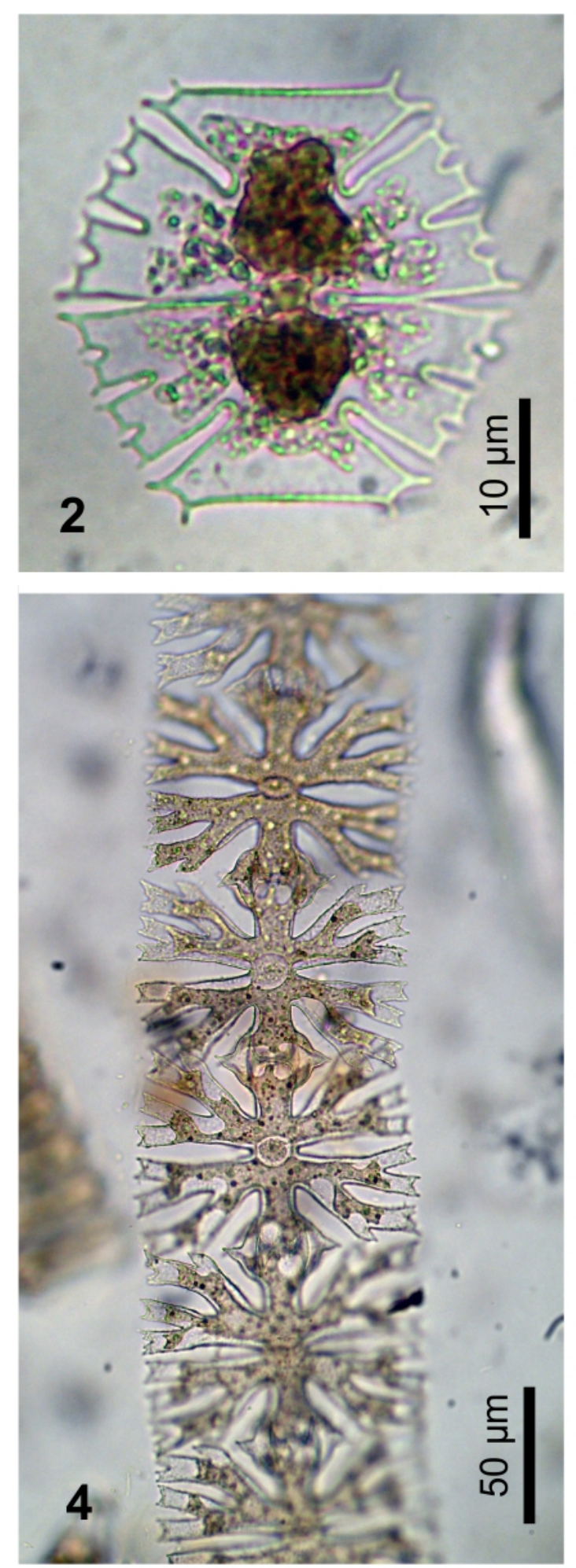

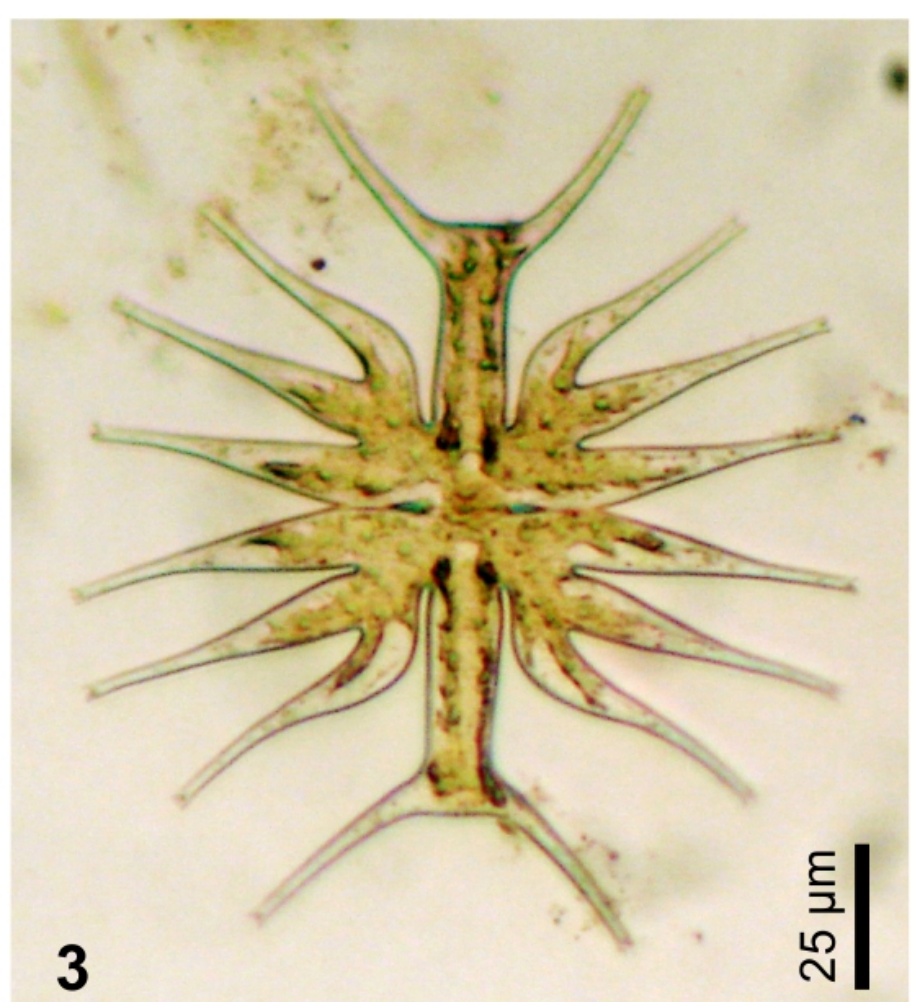

3

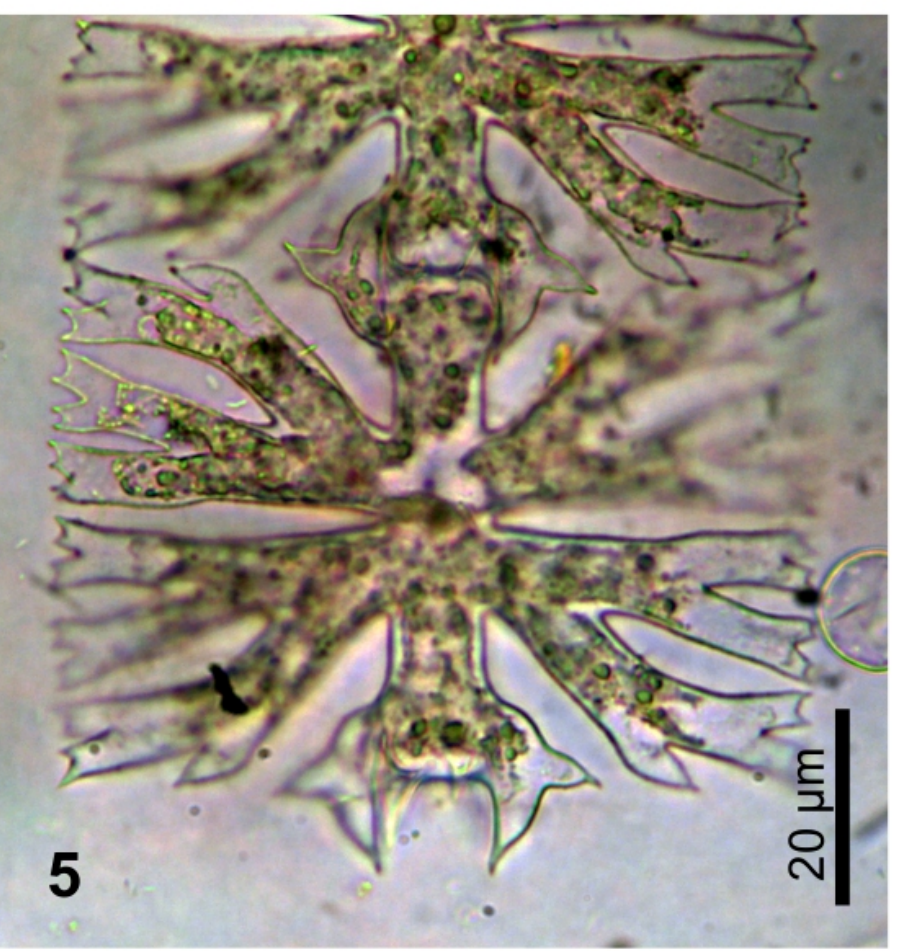

Figura 2. Micrasterias abrupta var. abrupta. 3. M. alata var. alata. 4, 5. M. foliacea var. foliacea.

Registros para o estado da Bahia. Bicudo \& Martins (1989), Oliveira et al. (2009), Ramos et al. (2011) e Oliveira (2011).

Oliveira (2011) e Ramos et al. (2011) relataram acentuado polimorfismo de Micrasterias furcata var. furcata f. furcata encontrada na planície litorânea e para a Serra da Jiboia, ambas no estado da Bahia. Os autores relataram a ocorrência de variações principalmente quanto às incisões interlobares e interlobulares, que ora se apresentavam mais rasas, com lobos polares mais curtos e mais largos na porção basal, podendo ser confundida com M. furcata var. pseudocrux (Grönblad) C.E.M.Bicudo \& Sormus, ora com incisões interlobulares e interlobares mais profundas, com o lobo polar relativamente mais estreito, de margem apical convexa e lóbulos mais delgados, se adequando à 
variedade típica. No material analisado, não foi constatado tal polimorfismo, estando de acordo com a descrição, medidas e ilustrações apresentadas por Bicudo \& Martins (1989).

Micrasterias laticeps Nordst. var. acuminata Willi Krieg in Rabenhorst, Krypt.-Fl. Deutschl. 13(2): 14; pl. 98, fig. 2. 1939.

Figura 8.

Célula ca. $90 \mu \mathrm{m}$ compr., ca. $115 \mu \mathrm{m}$ larg., istmo ca. $25 \mu \mathrm{m}$ larg., lobo polar ca. $120 \mu \mathrm{m}$ larg.

Material examinado - BRASIL. BAHIA: Andaraí, Marimbus do Baiano, 19 ago. 2011, C.W.N. Moura \& G.J.P. Ramos s.n. (HUEFS 185357); Lençóis, Marimbus do Remanso, 10 dez. 2011, C.A. Ribeiro \& G.J.P. Ramos s.n. (HUEFS 185402); ib., 11 fev. 2012, C.A. Ribeiro \& G.J.P. Ramos s.n. (HUEFS 185419).

Registros para o estado da Bahia. Oliveira et al. (2009) e Oliveira (2011).

Difere da variedade típica por apresentar as extremidades dos lobos basais acuminados. Bicudo \& Sormus (1982) e Oliveira et al. (2009) relataram variação morfológica quanto às margens apicais dos lobos polares, que ora apresentam-se levemente retusas no meio, ora uniformemente convexas, assim como às incisões interlobares, que apresentam-se com vértices acuminados ou levemente arredondados. Tais variações não foram observadas nos exemplares estudados.

Micrasterias laticeps Nordst. var. laticeps, Vidensk. Meddr dansk naturh. Foren. 1869(14-15): 220; pl. 2, fig. 14. 1869.

Figura 9.

Célula 100-115 $\mu \mathrm{m}$ compr., 137,5-150 $\mu \mathrm{m}$ larg., istmo 17,5-20 $\mu \mathrm{m}$ larg., lobo polar 127,5-137,5 $\mu \mathrm{m}$ larg.

Material examinado - BRASIL. BAHIA: Lençóis, Marimbus do Remanso, 2 abr. 2011, C.W.N. Moura \& G.J.P. Ramos s.n. (HUEFS 178358).

Registros para o estado da Bahia. Föster (1964, como Micrasterias laticeps Nordst. f. depressa Krieg. \& Scott), Bicudo \& Martins (1989), Oliveira et al. (2009) e Oliveira (2011).

Proposta por Nordstedt (1869) para material coletado em Minas Gerais, Micrasterias laticeps foi dividida, por esse autor, em duas formas, major e minor, baseadas tanto nas dimensões celulares quanto na atenuação dos lobos basais. A ilustração da f. major só foi publicada em Nordstedt (1887: pl. 2, fig. 14); a f. minor jamais foi ilustrada. Föster (1964) documentou e ilustrou Micrasterias laticeps Nordst. f. depressa Krieg. \& Scott, para o estado da Bahia, a qual difere da forma típica pela maior proporção na relação largura/comprimento. Entretanto, Bicudo \& Sormus (1982) sugeriram que a forma depressa é sinônimo da forma típica, considerando que a espécie engloba uma considerável variabilidade morfológica.
Micrasterias mahabuleshwarensis J.Hobson var. mahabuleshwarensis, Quart. J. Micr. Sc., ser. 2, 3: 169; fig. 1. 1863.

Figuras 10 e 11.

Célula 160-167,5 $\mu \mathrm{m}$ compr., 147-155 $\mu \mathrm{m}$ larg., istmo $22-30 \mu \mathrm{m}$ larg., lobo polar $80-85 \mu \mathrm{m}$ larg.

Material examinado - BRASIL. BAHIA: Andaraí, Marimbus do Baiano, 1 abr. 2011, C.W.N. Moura \& G.J.P. Ramos s.n. (HUEFS 178343); ib., 10 fev. 2012, C.A. Ribeiro \& G.J.P. Ramos s.n. (HUEFS 185409).

Registros para o estado da Bahia. Oliveira et al. (2009) e Oliveira (2011)

Oliveira et al. (2009) documentaram a ocorrência de Micrasterias mahabuleshwarensis pela primeira vez para a Bahia e destacaram a ausência de decoração supraistmal e de espinhos maiores na base da incisão entre os lobos basais e laterais. Estas características também estão ausentes nos exemplares estudados aqui. Embora as populações estudadas concordem em parte com a descrição e medidas apresentadas por Oliveira et al. (2009), diferem no que diz respeito à morfologia dos lobos laterais e basais, os quais são mais inflados e também por possuírem incisão interlobular mais profunda e aberta.

Micrasterias pinnatifida (Kütz.) Ralfs var. pinnatifida f. pinnatifida, Brit. Desm.: 77; pl. 10, fig. 3. 1848. Euastrum pinnatifidum Kütz., Phycol. Germ.: 134. 1845.

Figuras 12 e 13.

Célula 55-66,3 $\mu \mathrm{m}$ compr., 57-70 $\mu \mathrm{m}$ larg., istmo 7,5-12,5 $\mu \mathrm{m}$ larg., lobo polar 40-48,7 $\mu \mathrm{m}$ larg.

Material examinado - BRASIL. BAHIA: Andaraí, Marimbus do Baiano, 1 abr. 2011, C.W.N. Moura \& G.J.P. Ramos s.n. (HUEFS 178327); Lençóis, Marimbus do Remanso, 10 dez. 2011, C.A. Ribeiro \& G.J.P. Ramos s.n. (HUEFS 185398); ib., 10 fev. 2012, C.A. Ribeiro \& G.J.P.Ramos s.n. (HUEFS 185419).

Registros para o estado da Bahia. Bicudo \& Martins (1989), Oliveira et al. (2009) e Oliveira (2011).

De acordo com Sormus \& Bicudo (1974), $M$. pinnatifida é uma espécie bastante polimórfica e provavelmente as variedades taxonômicas podem não passar de expressões morfológicas da variedade-tipo. Nas populações estudadas, foram observadas variações morfológicas relativas aos dentículos das extremidades dos lobos polares e basais que se apresentaram ora 1denticulado, ora 2-dentículados. Růžička (1981) também relatou tais variações para o material da Europa.

Micrasterias radiosa Ralfs var. elegantior (G.S.West) Croasdale in Prescott et al., Syn. N. A. Desm. 2(2): 181; pl. 132, fig. 2-4. 1977. Micrasterias sol (Ehrenb.) Kütz. var. ornata Nordst. f. elegantior G.S.West, Mém. Soc. Neuchatel. Sci. Nat. 5: 1035; pl. 22, fig. 44. 1914.

Figuras 14 e 15.

Célula 185-207,5 $\mu \mathrm{m}$ compr., 175-202,5 $\mu \mathrm{m}$ larg., istmo $12,5-20 \mu \mathrm{m}$ larg., lobo polar 20,5-27,5 $\mu \mathrm{m}$ larg. 

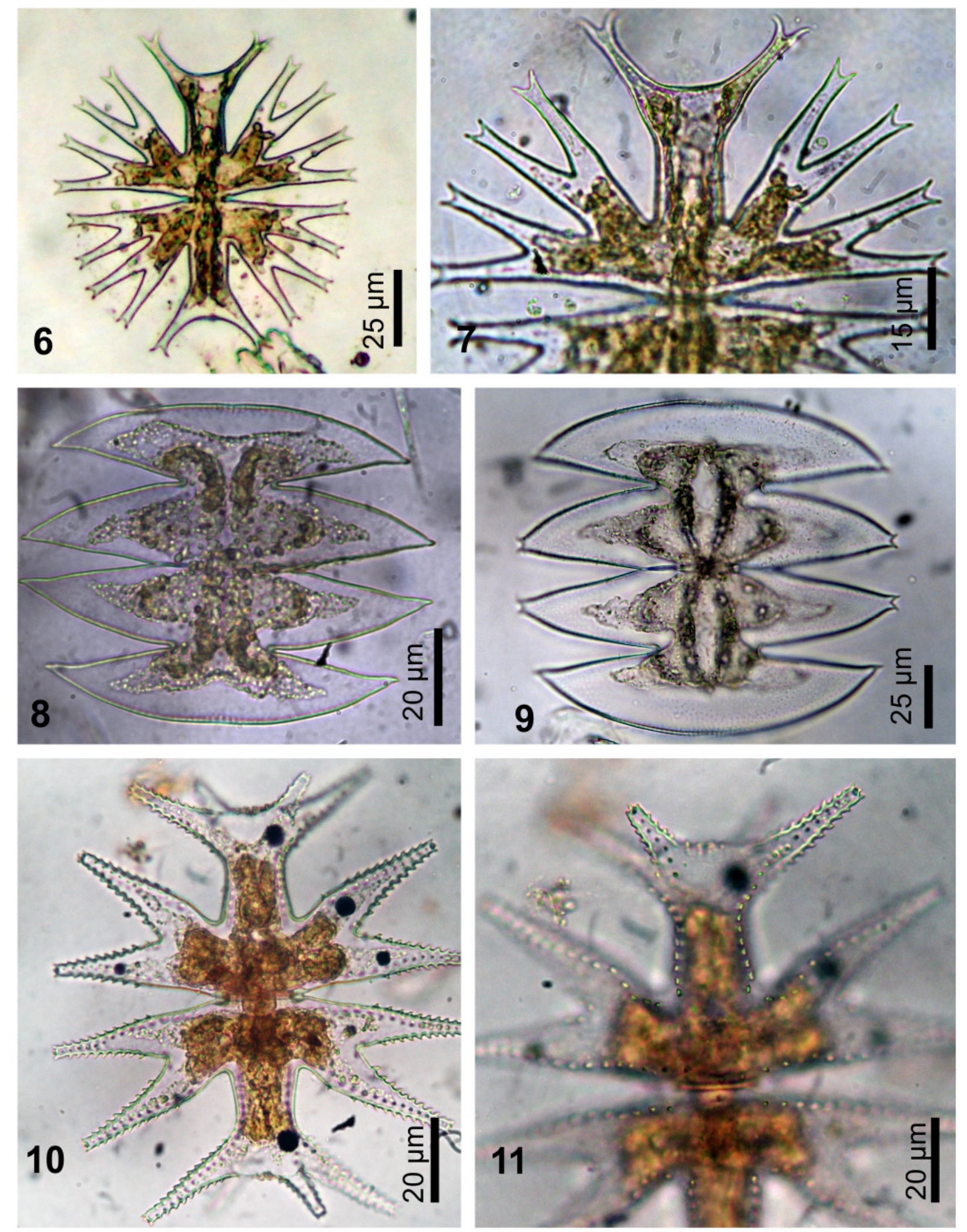

Figuras 6, 7. Micrasterias furcata var. furcata f. furcata. 8. M. laticeps var. acuminata. 9. M. laticeps var. laticeps. 10, 11. M. mahabuleshwarensis var. mahabuleshwarensis.

Material examinado - BRASIL. BAHIA: Andaraí, Marimbus do Baiano, 1 abr. 2011, C.W.N. Moura \& G.J.P. Ramos s.n. (HUEFS 178336); ib., 19 ago. 2011, C.W.N. Moura \& G.J.P. Ramos s.n. (HUEFS 185357); Lençóis, Marimbus do Remanso, 10 dez. 2011, C.A. Ribeiro \& G.J.P. Ramos s.n. (HUEFS 185403).
Registros para o estado da Bahia. Oliveira et al. (2009) e Oliveira (2011).

Micrasterias radiosa var. elegantior difere da variedade típica pelas incisões interlobares extremamente profundas, de forma que a parte central 
da semicélula é bastante reduzida e os lóbulos de todas as ordens fitáceos. Prescott et al. (1977) observaram a presença de espinhos ao longo das incisões maiores e destacaram que a variedade poderia ser confundida com M. radiosa var. ornata, que difere por apresenta uma fileira de espinhos ao longo de todas as incisões, ou pelo menos, das mais profundas. Bicudo \& Sormus (1982) registraram
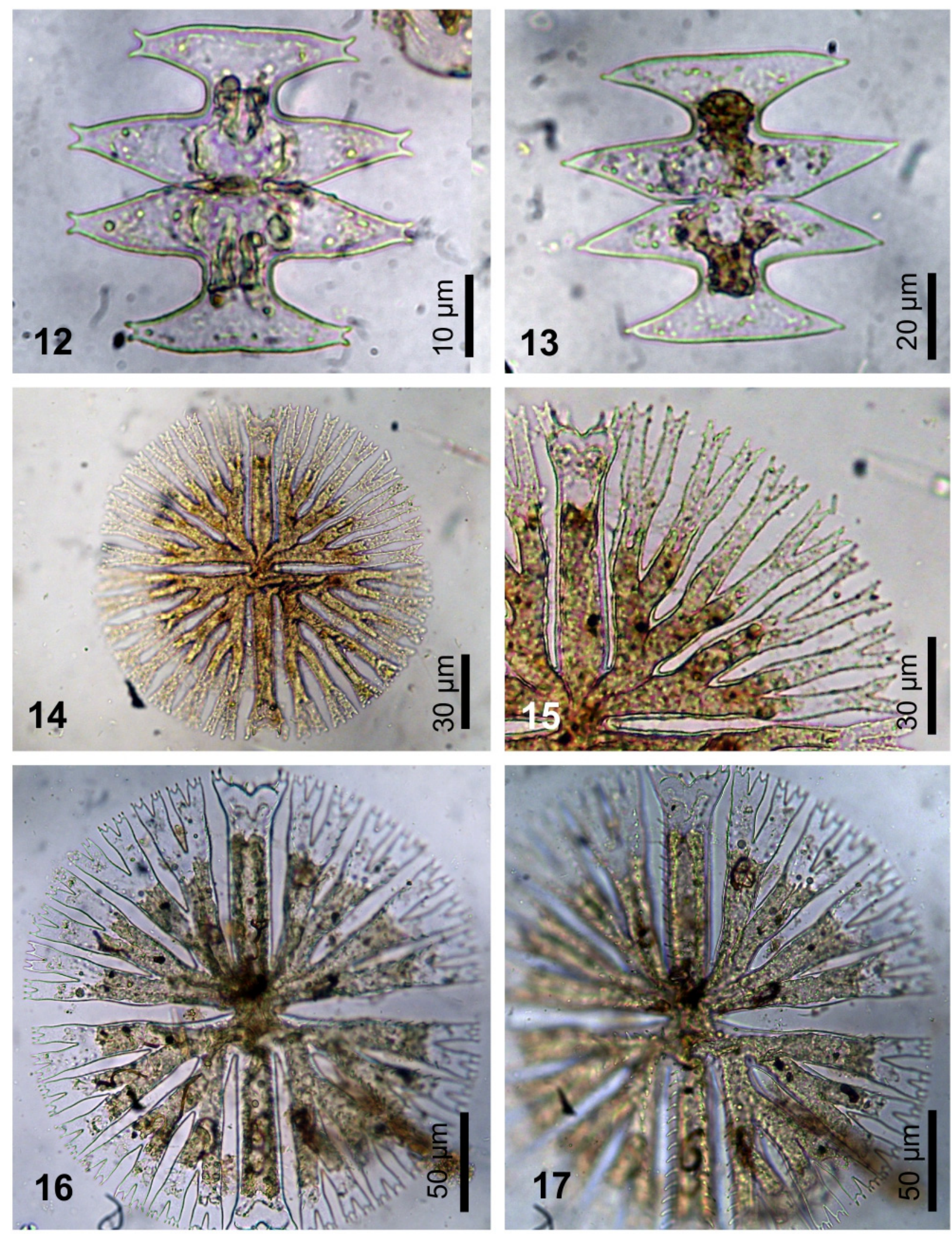

Figuras 12-13. Micrasterias pinnatifida var. pinnatifida f. pinnatifida. 14, 15. M. radiosa var. elegantior 16, 17. M. radiosa var. ornata f. aculeata. 
alguns exemplares com parede celular inteiramente glabra. No material analisado, foram encontrados exemplares portando espinhos ao longo das incisões interlobares e do seno mediano, como descrito por Prescott et al. (1977).

Micrasterias radiosa Ralfs var. ornata Nordst. f. aculeata (Willi Krieg.) Croasdale, Syn. N. A. Desm. 2(2): 183; pl. 131, fig.1-3. 1977. Micrasterias sol (Ehrenb.) Kütz. var. aculeata Willi Krieg. in Rabenhorst, Krypt.-Fl. Deutschl. 13(2): 94; pl. 131, fig. 2. 1939.

Figuras 16 e 17.

Célula 180-232 $\mu \mathrm{m}$ compr., 182-259 $\mu \mathrm{m}$ larg., istmo 17,5-25 $\mu \mathrm{m}$ larg., lobo polar 25-40 $\mu \mathrm{m}$ larg.

Material examinado - BRASIL. BAHIA: Andaraí, Marimbus do Baiano, 1 abr. 2011, C.W.N. Moura \& G.J.P. Ramos s.n. (HUEFS 178341); ib., 29 jun. 2011, C.A. Ribeiro \& G.J.P. Ramos s.n. (HUEFS 178372); Lençóis, Marimbus do Remanso, 1 abr. 2011, C.W.N. Moura \& G.J.P. Ramos s.n. (HUEFS 178358). (2011).

Registros para o estado da Bahia. Oliveira

Micrasterias radiosa var. ornata f. aculeata difere da forma típica da variedade por apresentar espinhos intramarginais mais longos, grosseiros, e numerosos ao longo das margens das incisões lobares e interlobulares. Pode ser confundida com $M$. schweinfurthii Cohn var. ornata Borge f. eckertii Kurt Först., porém esta última difere por apresentar contorno celular subcilíndrico, incisões interlobares menos profundas, lobo polar com a base levemente inflada e margem apical mais dilatada.

Micrasterias rotata (Grev.) Ralfs ex Ralfs var. rotata, Ann. Mag. Nat. Hist. 14: 259; pl. 6, fig. 1. 1844. Echinella rotata Grev., Diatomaceae in Dr. Hookers British Flora, Bd. 11. 1833.

Figura 18.

Célula 270-285 $\mu \mathrm{m}$ compr., 250-265 $\mu \mathrm{m}$ larg., istmo 35-37,5 $\mu \mathrm{m}$ larg., lobo polar 43,7-46,2 $\mu \mathrm{m}$ larg.

Material examinado - BRASIL. BAHIA: Andaraí, Marimbus do Baiano, 26 out. 2011, C.A. Ribeiro \& G.J.P. Ramos s.n. (HUEFS 185368); Lençóis, Marimbus do Remanso, 11 fev. 2012, C.A. Ribeiro \& G.J.P. Ramos s.n. (HUEFS 178358).

Registros para o estado da Bahia. Oliveira et al. (2009) e Oliveira (2011).

Segundo Lima (1982), a var. rotata é bastante característica pela morfologia e projeção dos lobos polares. Micrasterias rotata pode ser confundida com algumas expressões morfológicas de $M$. torreyi var. nordstedtiana, diferindo pela lobulação dos lobos laterais e basais. Bicudo \& Sormus (1982), ao analisarem várias populações, registraram polimorfismos relacionados à forma da porção distal dos lobos polares, projeção dos lobos polares, lobulação dos lobos basais e margem apical dos lóbulos de última ordem. Estas variações não foram observadas nas populações analisadas aqui.
Micrasterias simplex Börgesen var. minor Scott \& Croasd. in Scott et al., Acta Bot. Fenn. 69: 40; fig. 91. 1965.

Figura 19.

Célula ca. $30 \mu \mathrm{m}$ compr., ca. 22,5 $\mu \mathrm{m}$ larg., istmo ca. $10 \mu \mathrm{m}$ larg., lobo polar ca. $25 \mu \mathrm{m}$ larg.

Material examinado - BRASIL. BAHIA: Andaraí, Marimbus do Baiano, 26 out. 2011, C.A. Ribeiro \& G.J.P. Ramos s.n. (HUEFS 185376).

Referências para o estado da Bahia. Primeiro registro para o Estado (e para o Nordeste).

Segundo Bicudo \& Sormus (1982), essa variedade difere da típica por possuir dimensões menores, lobos basais mais arqueados para cima, seção transversal elíptica e seção lateral mais estreita acima da intumescência basal. O material analisado aqui está de acordo com a descrição, medidas e ilustração apresentadas por Scott et al. (1965), a partir de material coletado em depressões de campo inundado em Vigia, Pará, e por Bicudo \& Sormus (1982) para o material de São Carlos, São Paulo.

Micrasterias torreyi Bailey ex Ralfs var. nordstedtiana (Hieronymus) Schmidle, Bot. Jahrb. 26(1-2): 48. 1898. Micrasterias nordstedtiana Hieronymus, Pflanzenwelt Öst-Afrikas 1: 21. 1895.

Figura 20.

Célula 260-270 $\mu \mathrm{m}$ compr., 250-260 $\mu \mathrm{m}$ larg., istmo 22,5-27 $\mu \mathrm{m}$ larg., lobo polar 47,5-60 $\mu \mathrm{m}$ larg.

Material examinado - BRASIL. BAHIA: Andaraí, Marimbus do Baiano, 1 abr. 2011, C.W.N. Moura \& G.J.P. Ramos s.n. (HUEFS 178336); Lençóis, Marimbus do Remanso, 2 abr. 2011, C.W.N. Moura \& G.J.P. Ramos S.n. (HUEFS 178358).

Registros para o estado da Bahia. Oliveira (2011).

O primeiro registro de $M$. torreyi var. nordstedtiana para a Bahia foi noticiado por Oliveira (2011) para a APA Litoral Norte do Estado. A variedade nordstedtiana difere da típica por apresentar células um pouco mais longas que largas, lobos bidenticulados e base dos lobos com as margens internas infladas, recobrindo parcialmente uns aos outros. As populações analisadas concordam com a descrição, medidas e ilustrações apresentadas por Bicudo \& Sormus (1982) para o estado de São Paulo e por Oliveira (2011) para a Bahia. No entanto, o polimorfismo relatado por eles para a lobulação dos lobos laterais e basais não foi encontrado nos exemplares estudados aqui.

Micrasterias tropica Nordst. var. tropica, Vid. Medd. Naturh. For. Kjobenhavn 21: 219; pl. 2, fig. 15. 1870.

Figuras 21 e 22.

Célula 110-121 $\mu \mathrm{m}$ compr., 93-100 $\mu \mathrm{m}$ larg., istmo 15-18 $\mu \mathrm{m}$ larg., lobo polar 40-51,2 $\mu \mathrm{m}$ larg.

Material examinado - BRASIL. BAHIA: Andaraí, Marimbus do Baiano, 1 abr. 2011, C.W.N. Moura \& G.J.P. Ramos s.n. (HUEFS 178329); Lençóis, Marimbus do Remanso, 2 abr. 2011, C.W.N. Moura \& G.J.P. Ramos s.n. (HUEFS 178347). 

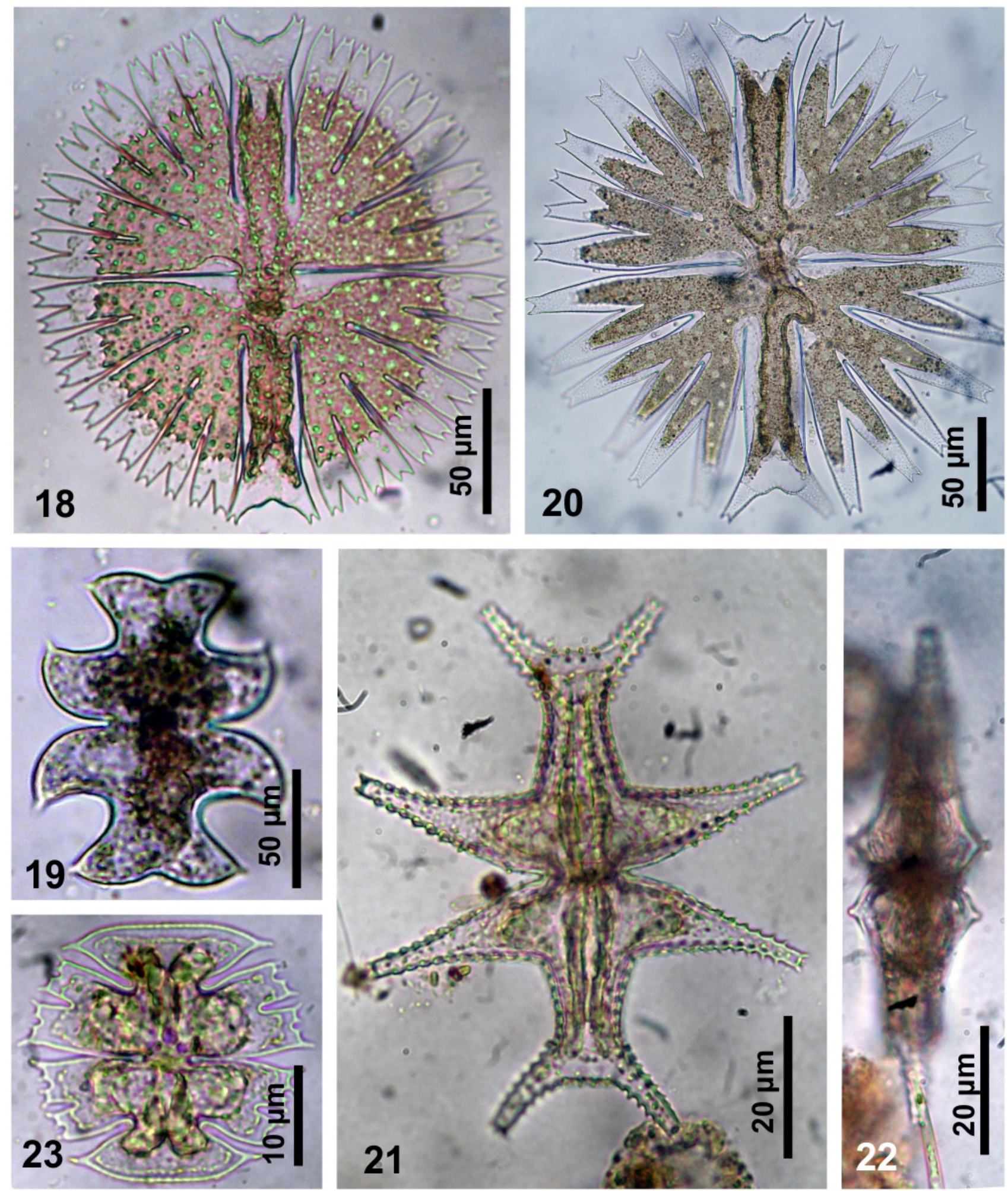

Figura 18. Micrasterias rotata var. rotata. 19. M. simplex var. minor. 20. M. torreyi var. nordstedtiana. 21, 22. M. tropica. var. tropica: 21- vista frontal; 22- vista lateral. 23. M. truncata var. pusilla.

Registros para o estado da Bahia. Primeiro registro para o Estado (e para o Nordeste).

A forma do lobo polar e a ornamentação da parede celular são semelhantes às de M. mahabuleshwarensis, que difere quanto às dimensões celulares e por possuir lobos laterais. Ainda quanto à ornamentação celular, ela lembra $M$. hardyi, que se distingue pelas dimensões da célula, forma e tamanho do lobo polar. O material estudado aqui está de acordo com a descrição e medidas de Bicudo \& Sormus (1982), mas difere das ilustrações quanto à disposição dos lobos basais, que são levemente voltados para cima nas populações dos 
Marimbus. Bicudo \& Sormus (1982) apontaram a necessidade de revisão taxonômica dos táxons $M$. hardyi, M. mahabuleshwarensis e categorias infraespecíficas de M. tropica.

Micrasterias truncata (Corda) Bréb. ex Ralfs var. pusilla G.S.West, Mém. Soc. Neuchat. Sci. Nat., ser. 2, 5: 1035; pl. 22, fig. 42-43. 1914. Cosmarium truncatum Corda, Alman. Carlsbad 9: 243. 1839.

Figura 23.

Célula 38,7-42,5 $\mu \mathrm{m}$ compr., 47,5-57,5 $\mu \mathrm{m}$ larg., istmo 7,5-12,5 $\mu \mathrm{m}$ larg., lobo polar 31,2-40 $\mu \mathrm{m}$ larg.

Material examinado - BRASIL. BAHIA: Andaraí, Marimbus do Baiano, 1 abr. 2011, C.W.N. Moura \& G.J.P. Ramos s.n. (HUEFS 78332); Lençóis, Marimbus do Remanso, 2 abr. 2011, C.W.N. Moura \& G.J.P. Ramos s.n. (HUEFS 178360).

Registros para o estado da Bahia. Oliveira et al. (2009) e Oliveira (2011).

Difere da variedade típica por apresentar tamanho relativamente reduzido, células um pouco mais largas que longas, ângulos dos lobos polares acuminados e incisões entre os lobos polares e basais mais profundas. Bicudo \& Sormus (1982) ressaltam o grande polimorfismo da variedade, que pode ser confundida com M. decemdentata (Näg.) Arch., da qual difere por apresentar lobos polares um pouco mais curtos, e com M. abrupta, diferindo pelo seno mediano linear e fechado. As populações analisadas aqui apresentaram grande variação morfológica quanto à forma do lobo polar, que ora apresentou-se levemente convexo, ora reto com um ou dois dentículos na extremidade, e quanto à morfologia dos lobos laterais e basais, que ora apresentou-se com incisões mais curtas, concordando com o descrito por Felisberto \& Rodrigues (2011) para o material coletado na bacia do rio Paranapanema, Paraná/São Paulo. Tal polimorfismo tornou a identificação um pouco problemática, sendo necessários estudos mais detalhados para avaliar se essas variações morfológicas podem ser atribuídas apenas a um táxon, como sugeriram Bicudo \& Sormus (1982). Bicudo \& Martins (1989) e Oliveira et al. (2009) referiram exemplares maiores que dos Marimbus.

\section{DISCUSSÃO}

A partir das 120 unidades amostrais obtidas durante os períodos seco e chuvoso foram inventariados 15 táxons de Micrasterias para o Pantanal dos Marimbus, dos quais 11 foram comuns às duas áreas estudadas, três exclusivos do Marimbus do Baiano (Micrasterias furcata, M. mahabuleshwarensis e M. simplex var. minor) e um restrito ao Marimbus do Remanso ( $M$. laticeps var. laticeps) (Tabelas 1 e 2). Dos 14 táxons de Micrasterias encontrados no Marimbus do Baiano, 11 ocorreram nos dois períodos, sendo $M$. pinnatifida var. pinnatifida a espécie mais frequente ( $>90 \%$ ). Micrasterias tropica var. tropica apresentou baixa frequência de ocorrência tanto no período seco quanto no chuvoso $(<30 \%$ das amostras). Dentre os táxons inventariados no Marimbus do Baiano, apenas $M$. simplex var. minor foi considerado exclusivo do período chuvoso, enquanto $M$. foliacea var. foliacea, M. laticeps var. acuminata e $M$. torreyi var. nordstedtiana apareceram exclusivamente no período seco (Tabela 1). Dos 12 táxons de Micrasterias do Marimbus do Remanso, M. truncata var. pusilla foi o mais frequente $(>85 \%)$. Micrasterias foliacea var. foliacea, M. laticeps var. acuminata e $M$. rotata var. rotata foram exclusivos do período chuvoso e $M$. abrupta var. abrupta, M. alata var. alata, M. laticeps var. laticeps, M. torreyi var. nordstedtiana, $M$. radiosa var. ornata f. aculeata foram exclusivos do período seco (Tabela 1).

A partir do cálculo de frequência relativa global dos táxons de Micrasterias inventariados no Pantanal dos Marimbus (Tabela 2), constatou-se que $M$. pinnatifida e M. truncata var. pusilla foram muito frequentes no Marimbus do Baiano e M. foliacea, M. laticeps var. acuminata, M. mahabuleshwarensis, M. radiosa var. ornata f. aculeata, $M$. rotata var. rotata e $M$. simplex var. minor foram raros. Já no Marimbus do Remanso, verificou-se que a maioria dos táxons (10) teve ocorrência rara, com predomínio de $M$. truncata var. pusilla, com frequência relativa superior a $91 \%$ das amostras, sendo um táxon classificado como muito frequente. Ao comparar os dados obtidos neste estudo com os de trabalhos de levantamento florístico na Bahia, observou-se uma discrepância quanto ao número de táxons inventariados. Föster (1964) listou três táxons de Micrasterias a partir de coletas realizadas na região da Chapada Diamantina (Pico das Almas e Rio de Contas) e Ramos et al. (2011) descreveram apenas dois táxons a partir de coletas realizadas em ambientes fitotelmatas bromelícola na Serra da Jiboia. O menor número de táxons encontrado por Ramos et al. (2011) possivelmente está relacionado ao ambiente extremamente restritivo onde aquele estudo foi realizado. No caso de Foster (1964), o número reduzido de táxons pode ter sido causado por subamostragem, já que ele se baseou em amostras obtidas por coletores não especializados.

Confrontando os resultados obtidos aqui com os dos trabalhos na região litorânea do Estado, percebe-se que o número de táxons foi similar ao encontrado por Oliveira et al. (2009), que inventariaram 14 táxons de Micrasterias para duas áreas de Proteção Ambiental (APA Capivara e APA Lagoas de Guarajuba) Litoral Norte da Bahia, porém inferior aos dados de Oliveira (2011), que registrou 28 táxons para a planície litorânea, Norte da Bahia. A diferença de táxons em relação a este último pode estar relacionada à grande diversidade de corpos aquáticos estudados ao longo da planície litorânea. Apesar de Martins \& Bicudo (1987) e Bicudo \& Martins (1989) também terem citado táxons de Micrasterias para a planície litorânea do Estado, as riquezas de táxons encontradas por eles foram inferiores às obtidas no presente estudo. 
Tabela 1. Ocorrência (x) nos períodos seco (abril, junho e agosto de 2011) e chuvoso (outubro e dezembro de 2011 e fevereiro de 2012), frequência de ocorrência absoluta (FA) e frequência de ocorrência relativa (FR) dos táxons de Micrasterias amostrados no Pantanal dos Marimbus (Baiano e Remanso), Chapada Diamantina, Bahia, Brasil.

\begin{tabular}{|c|c|c|c|c|c|c|c|c|c|c|c|c|c|c|c|c|c|c|c|c|}
\hline \multirow{3}{*}{ Táxons } & \multicolumn{10}{|c|}{ Marimbus Baiano } & \multicolumn{10}{|c|}{ Marimbus Remanso } \\
\hline & \multicolumn{5}{|c|}{ Período Seco } & \multicolumn{5}{|c|}{ Período Chuvoso } & \multicolumn{5}{|c|}{ Período Seco } & \multicolumn{5}{|c|}{ Período Chuvoso } \\
\hline & Abr. & Jun. & Ago. & FA & FR & Out. & Dez. & Fev. & FA & FR & Abr. & Jun. & Ago. & FA & FR & Out. & Dez. & Fev. & FA & FR \\
\hline M. abrupta & $\mathrm{X}$ & $\mathrm{X}$ & - & 6 & 13,3 & $\mathrm{X}$ & $\mathrm{X}$ & $\mathrm{X}$ & 12 & 23,3 & $\mathrm{X}$ & - & - & 1 & 3,3 & - & - & - & - & - \\
\hline M. alata & $\mathrm{X}$ & $\mathrm{X}$ & $\mathrm{X}$ & 96 & 66,7 & $\mathrm{X}$ & $\mathrm{X}$ & $\mathrm{X}$ & 90 & 50 & $\mathrm{X}$ & - & - & 4 & 3,3 & - & - & - & - & - \\
\hline M. foliacea & $\mathrm{X}$ & - & - & 5 & 10 & - & - & - & - & - & - & - & - & - & - & - & $\mathrm{X}$ & - & 1 & 3,3 \\
\hline M. furcata & $\mathrm{X}$ & $\mathrm{X}$ & $\mathrm{X}$ & 55 & 50 & $\mathrm{X}$ & $\mathrm{X}$ & $\mathrm{X}$ & 23 & 43,3 & - & - & - & - & - & - & - & - & - & - \\
\hline $\begin{array}{l}\text { M. laticeps } \\
\text { var. acuminata }\end{array}$ & - & - & $\mathrm{X}$ & 1 & 3,3 & - & - & - & - & - & - & - & - & - & - & - & $\mathrm{X}$ & $\mathrm{X}$ & 5 & 13,3 \\
\hline $\begin{array}{l}\text { M. laticeps } \\
\text { var. laticeps }\end{array}$ & - & - & - & - & - & - & - & - & - & - & $\mathrm{X}$ & - & - & 2 & 3,3 & - & - & - & - & - \\
\hline $\begin{array}{l}\text { M. mahabuleshwarensis } \\
\text { var.mahabuleshwarensis }\end{array}$ & $\mathrm{X}$ & - & - & 1 & 3,3 & - & - & $\mathrm{X}$ & 1 & 3,3 & - & - & - & - & - & - & - & - & - & - \\
\hline M. pinnatifida & $\mathrm{X}$ & $\mathrm{X}$ & $\mathrm{X}$ & 436 & 93,3 & $\mathrm{X}$ & $\mathrm{X}$ & $\mathrm{X}$ & 530 & 100 & $\mathrm{X}$ & $\mathrm{X}$ & $\mathrm{X}$ & 21 & 20 & - & $\mathrm{X}$ & $\mathrm{X}$ & 19 & 33,3 \\
\hline $\begin{array}{l}\text { M. radiosa } \\
\text { var. elegantior }\end{array}$ & $\mathrm{X}$ & $\mathrm{X}$ & $\mathrm{X}$ & 21 & 33,3 & $\mathrm{X}$ & $\mathrm{X}$ & $\mathrm{X}$ & 8 & 16,7 & $\mathrm{X}$ & - & - & 2 & 6,7 & - & $\mathrm{X}$ & $\mathrm{X}$ & 4 & 13,3 \\
\hline $\begin{array}{l}\text { M. radiosa var. ornata } \\
\text { f. aculeata }\end{array}$ & $\mathrm{X}$ & $\mathrm{X}$ & $\mathrm{X}$ & 3 & 10 & - & $\mathrm{X}$ & - & 3 & 6,7 & $\mathrm{X}$ & - & - & 2 & 6,7 & - & - & - & - & - \\
\hline M. rotata var. rotata & - & $\mathrm{X}$ & - & 1 & 3,3 & $\mathrm{X}$ & - & - & 3 & 3,3 & - & - & - & - & - & - & - & $\mathrm{X}$ & 1 & 3,3 \\
\hline M. simplex var. minor & - & - & - & - & - & $\mathrm{X}$ & - & - & 2 & 6,7 & - & - & - & - & - & - & - & - & - & - \\
\hline $\begin{array}{l}\text { M. torreyi } \\
\text { var. nordstedtiana }\end{array}$ & $\mathrm{X}$ & $\mathrm{X}$ & - & 9 & 16,7 & - & - & - & - & - & $\mathrm{X}$ & - & - & 2 & 3,3 & - & - & - & - & - \\
\hline M. tropica var. tropica & $\mathrm{X}$ & $\mathrm{X}$ & $\mathrm{X}$ & 12 & 30 & $\mathrm{X}$ & - & $\mathrm{X}$ & 3 & 10 & $\mathrm{x}$ & $\mathrm{X}$ & - & 23 & 26,7 & $\mathrm{X}$ & $\mathrm{X}$ & $\mathrm{X}$ & 8 & 20 \\
\hline M. truncata var. pusilla & $X$ & $X$ & $\mathrm{X}$ & 330 & 80 & $X$ & $X$ & $X$ & 242 & 86,6 & $\mathrm{X}$ & $\mathrm{X}$ & $\mathrm{X}$ & 295 & 86,6 & $X$ & $X$ & $X$ & 976 & 96,6 \\
\hline
\end{tabular}

Tabela 2. Frequência absoluta (FA) e frequência de ocorrência relativa (FR) globais e categorias (C) dos táxons de Micrasterias amostrados no Pantanal dos Marimbus (Baiano e Remanso), Chapada Diamantina, Bahia, Brasil. F- frequente; MF- muito frequente; PF- pouco frequente; R- rara.

\begin{tabular}{|c|c|c|c|c|c|c|}
\hline \multirow{3}{*}{ Táxons } & \multicolumn{6}{|c|}{ Frequência Global } \\
\hline & \multicolumn{3}{|c|}{ Marimbus Baiano } & \multicolumn{3}{|c|}{ Marimbus Remanso } \\
\hline & FA & FR & $\mathrm{C}$ & FA & FR & $\mathrm{C}$ \\
\hline M. abrupta & 18 & 18,3 & $\mathrm{PF}$ & 1 & 1,7 & $\mathrm{R}$ \\
\hline M. alata & 186 & 58,3 & $\mathrm{~F}$ & 4 & 1,7 & $\mathrm{R}$ \\
\hline M. foliacea & 5 & 5,0 & $\mathrm{R}$ & 1 & 1,7 & $\mathrm{R}$ \\
\hline M. furcata & 78 & 46,7 & $\mathrm{~F}$ & - & - & - \\
\hline M. laticeps var. acuminata & 1 & 1,7 & $\mathrm{R}$ & 5 & 6,7 & $\mathrm{R}$ \\
\hline M. laticeps var. laticeps & - & - & - & 2 & 1,7 & $\mathrm{R}$ \\
\hline M. mahabuleshwarensis var. mahabuleshwarensis & 2 & 3,3 & $\mathrm{R}$ & - & - & - \\
\hline M. pinnatifida & 966 & 96,7 & MF & 40 & 28,3 & $\mathrm{PF}$ \\
\hline M. radiosa var. elegantior & 29 & 25,0 & $\mathrm{PF}$ & 6 & 10,0 & $\mathrm{R}$ \\
\hline M. radiosa var. ornata f. aculeata & 6 & 8,3 & $\mathrm{R}$ & 2 & 3,3 & $\mathrm{R}$ \\
\hline M. rotata var. rotata & 4 & 5,0 & $\mathrm{R}$ & 1 & 1,7 & $\mathrm{R}$ \\
\hline M. simplex var. minor & 2 & 3,3 & $\mathrm{R}$ & - & - & - \\
\hline M. torreyi var. nordstedtiana & 9 & 8,3 & $\mathrm{R}$ & 2 & 1,7 & $\mathrm{R}$ \\
\hline M. tropica var. tropica & 15 & 20,0 & $\mathrm{PF}$ & 31 & 18,3 & $\mathrm{PF}$ \\
\hline M. truncata var. pusilla & 572 & 81,7 & MF & 1271 & 91,7 & MF \\
\hline
\end{tabular}


Com exceção de Micrasterias laticeps var. laticeps, todos os demais táxons tiveram o conhecimento da distribuição geográfica ampliada para a Chapada Diamantina. Além disso, Micrasterias tropica Nordst. var. tropica e $M$. simplex var. minor são adições à flora ficológica do Nordeste do Brasil.

\section{REFERÊNCIAS}

Bicudo, C.E.M. \& Sormus, L. 1982. Desmidiofórula Paulista II: gênero Micrasterias C.Agardh ex Ralfs. Bibliotheca Phycologica 57: 1-230.

Bicudo, C.E.M. \& Martins, D.V. 1989. Desmídias (Zygnemaphyceae) de Itanagra, estado da Bahia, Brasil. Revista Brasileira de Biologia 49(1): 309-324.

Bicudo, C.E.M. \& Menezes, M. 2006. Gêneros de Algas de Águas Continentais do Brasil: chave para identificação e descrições. 2 ed. RiMa, São Carlos.

Felisberto, S. \& Rodrigues, L. 2011. Desmídias epifíticas do reservatório de Rosana, bacia do rio Paranapanema, Paraná/São Paulo, Brasil: Euastrum e Micrasterias (Desmidiaceae). Revista Brasileira de. Biociências 9(2): 206-213.

Förster, K. 1964. Desmidiaceen aus Brasilien, 2, Teil: Bahia, Goyaz, Piauhy und Nord-Brasilien. Hydrobiologia 22(3-4): 321-505.

França, F.; Melo, E.; Oliveira, I.B.; Reis, A.T.C.C.; Alves, G.L. \& Costa, M.F. 2010. Plantas vasculares das áreas alagadas dos Marimbus, Chapada Diamantina, Bahia, Brasil. Hoehnea 37(4): 719-730.

Funch, R. 2002. Um Guia para a Chapada Diamantina. 3 ed. Nova Civilização, Cruz das Almas.

Gerrath, J.F. 2003. Conjugating green algae and desmids. In: J.D. Wehr \& R.G. Sheath (eds), Freshwater Algae of North America: ecology and classification. Elsevier Science Academic Press, San Diego, p. 353-381.

Guiry, M.D. \& Guiry, G.M. 2014. AlgaeBase. World-wide electronic publication, National University of Ireland, Galway. Disponível em http://www.algaebase.org; acesso em 2 jul. 2014.

Lima, M.G.S.M. 1982. Desmidiaceae (Zygnemaphyceae) do Município do Rio de Janeiro e Arredores: uma contribuição ao seu conhecimento. Dissertação de Mestrado. Universidade Federal do Rio de Janeiro.

Lima, C.C.U. \& Nolasco, M.C. 1997. Lençóis: uma ponte entre a geologia e o homem. Departamento de Ciências ExatasUEFS, Feira de Santana.

Martins, D.V. \& Bicudo, C.E.M. 1987. Desmídias da Ilha de Itanharé, estado da Bahia, Brasil. Revista Brasileira de Biologia 47(1-2): 1-16.
Matteucci, S.D. \& Colma, A. 1982. Metodologia para el Estudo de la Vegetacion. OEA/PRDECT, Washington.

Nordstedt, O. 1869. Desmidiaceae. Symbolae ad floram Brasiliae centralis cognoscendam, 5. Videnskabelige Meddelelser fra Dansk Naturhistorisk Forening i Kjøbenhavn 1869(14-15): 195-234.

Nordstedt, C.F.O. 1887. Algologiska smasaker, 4: Utdrag ur ett arbet öfver de af Dr. S. Berggren pa Nya Seland och in Australien samlade sötvattensalgerna. Botaniska Notiser 1887: $153-164$.

Oliveira, I.V.B. 2011. Zygnematophyceae (Streptophyta) da Área de Proteção Ambiental Litoral Norte, Bahia, Brasil. Tese de Doutorado. Universidade Estadual de Feira de Santana.

Oliveira, I.B.; Moura, C.W.N. \& Bicudo, C.E.M. 2009. Micrasterias C. Agardh ex Ralfs (Zygnematophyceae) de duas Áreas de Proteção Ambiental da planície litorânea do norte da Bahia, Brasil. Revista Brasileira de Botânica 32(2): 213-232.

Prescott, G.W.; Croasdale H.T. \& Vinyard W.C. 1977. A Synopsis of North American desmids. Part II: Desmidiaceae: Placodermae. Section 2. University of Nebraska Press, Lincoln, London.

Ramos, G.J.P.; Oliveira, I.B. \& Moura, C.W.N. 2011. Desmídias de ambiente fitotelmata bromelícola da Serra da Jiboia, Bahia, Brasil. Revista Brasileira de Biociências 9(1): 103-113.

Ramos, G.J.P.; Bicudo, C.E.M.; Góes-Neto, A. \& Moura, C.W.N. 2012. Monoraphidium and Ankistrodesmus (Chlorophyceae, Chlorophyta) from Pantanal dos Marimbus, Chapada Diamantina, Bahia State, Brazil. Hoehnea 39(3): 421-434.

Ramos, G.J.P.; Bicudo, C.E.M.; Góes-Neto, A. \& Moura, C.W.N. 2014. New additions of coccoid green algae to the phycoflora of Brazil and the Neotropics. Acta Botanica Brasilica 28(1): 8-16.

Růžička, J. 1981. Die Desmidiaceen Mitteleuropas. Vol. 1(2). E. Schweizerbart'sche Verlagsbuchhandlung, Stuttgart.

Santana, L.M. 2011. Análise Física e Química da Água e Estrutura da Comunidade Fitoplanctônica do Rio Almada Sul da Bahia. Dissertação de Mestrado. Universidade Estadual de Santa Cruz.

Scott, A.M.; Grönblad, R. \& Croasdale, H.T. 1965. Desmids from the Amazon basin, Brasil, collected by Dr. H. Sioli. Acta Botanica Fennica 69: 1-94

SEMA (Secretaria do Meio Ambiente - Governo do Estado da Bahia) 2012. Disponível em http://www.semarh.ba.gov.br/ conteudo.aspx?s=APAMARIM $\&$ p=APAAPA; acesso em 29 abr. 2012.

Sormus, L. \& Bicudo, C.E.M. 1974. Polymorphism in the desmid Micrasterias pinnatifida and its taxonomical implications. Journal of Phycology 10: 274-279. 